\title{
Clustering and Prediction Analysis of the Coordinated Development of China's Regional Economy Based on Immune Genetic Algorithm
}

\author{
Yang Yang \\ Chengdu Wuhou District Xueyou Education Training School, Chengdu 610000, China \\ Correspondence should be addressed to Yang Yang; william115@pku.edu.cn
}

Received 7 January 2021; Revised 4 February 2021; Accepted 24 February 2021; Published 2 March 2021

Academic Editor: Wei Wang

Copyright ( 2021 Yang Yang. This is an open access article distributed under the Creative Commons Attribution License, which permits unrestricted use, distribution, and reproduction in any medium, provided the original work is properly cited.

\begin{abstract}
Since the opening of the economy, China's regional economy has developed rapidly, the overall national strength has been increasing, and the people's living standards have been continuously improved. The issue of coordinated regional development has become an important issue in today's society. Genetic algorithm is a kind of prediction algorithm that has developed rapidly in recent years and is widely used. However, when solving engineering prediction problems, there are often problems such as premature convergence and easiness to fall into local optimal solutions. Therefore, on the basis of studying the related theories of genetic algorithm and artificial immune algorithm, this paper uses the advantages of the two algorithms, combines the two algorithms, and proposes an improved algorithm for genetic algorithm-adaptive immune genetic algorithm. Taking genetic algorithm as the basic framework, the operators and selection methods of artificial immune algorithm are integrated. Using the adaptive concept, the formulas of adaptive crossover probability and mutation probability are innovatively designed. Compared with the fixed value of the immune genetic algorithm, the introduction of the adaptive concept can intelligently adjust the optimization process and increase the optimization speed. Considering the double uncertain factors of product market demand and waste product recycling in the remanufacturing supply chain system, the maximization of logistics network operating profit, the minimization of environmental impact, and the maximization of customer satisfaction are the forecast goals. The market demand of uncertain products is effectively controlled through the option contract mechanism, and a multiobjective forecasting model based on the option contract mechanism is established. According to the characteristics of the model, an improved immune genetic algorithm is designed to solve the problem, and the effectiveness of the immune genetic algorithm is verified through an example.
\end{abstract}

\section{Introduction}

Regional development issues are no longer purely economic issues. How to improve economic quality while maintaining the growth momentum of economic development, highlighting the concept of fairness and justice, and ensuring and improving the people's well-being are important demands of today's social development [1-3]. As an important part of shared development, basic public services are closely related to people's lives, and achieving equal development is an important yardstick for measuring people's shared development results. The high-quality basic public service level allows people to have more livelihood security, and they can reduce the phenomenon of passive savings without much worry. Although China's public service system continues to improve, as the people's requirements for public services are getting higher and higher, shortcomings such as insufficient scale of facilities, low service quality, and uneven distribution of regional resources and development have gradually become prominent $[4,5]$. The level of economic development weakens citizens' sense of gain and happiness, which is not conducive to building a fair and just social environment [6].

Compared with the traditional supply chain system, the remanufacturing supply chain system not only includes the traditional positive product sales supply chain but also 
extends to the reverse waste product recycling supply chain, thus forming a closed-loop supply chain system. Therefore, the logistics network design problem of the remanufacturing supply chain system includes not only the forward product sales logistics network design but also the reverse waste product recycling logistics network design. In addition, in the study of the logistics network design of the remanufacturing supply chain, it is necessary to take into account the double uncertainties of the positive product market demand and the reverse recycling of waste products. In a typical study on the joint supplement of multiple spare parts, only the positive joint supplement of multiple spare parts is considered. However, for the joint replenishment of multiple spare parts in the remanufacturing supply chain system, in addition to the forward joint replenishment of multiple spare parts, it is also necessary to consider the joint recovery of reverse used spare parts. In addition, for the joint replenishment of multiple spare parts in the remanufacturing supply chain system, not only must the deterministic joint replenishment problem be optimized, but also the random joint replenishment problem must be studied.

The researchers analyzed a multistage inventory system that obeys Markov demand [7, 8]. The demand distribution of each stage is determined by the current state of an exogenous Markov chain. The extra demand is backlogged, and the linear holding cost stages exist, linear reordering cost exists in the first stage, and the ordering cost is also linear [9]. The goal is to minimize the long-term average cost of the system. The results show that the optimal strategy is a multistage, basic inventory ordering strategy with interdependent states. An effective algorithm is proposed to determine the optimal basic inventory level, and the result can be extended to a fixed ordering cost in the Nth stage continuous systems and integrated systems with linear ordering costs. Relevant scholars analyzed a supply chain inventory management strategy based on the concept of multilevel inventory and fuzzy set theory and used a multilevel inventory model to manage supply chain inventory in an overall manner, treating market demand, and holding costs $[10,11]$. Set theory is used to establish an uncertainty model related to market demand and inventory costs (including holding costs and delay costs) and then apply this theory to a three-stage supply chain to show its feasibility [12]. Finally, through simulation, it is proved that the three-stage supply chain is better than the local safety stock management strategy. Relevant scholars construct an indicator system from four dimensions and use the network analytic method and the urban-rural ratio method to analyze and study the sharing index of each city $[13,14]$. The analysis found that the degree of shared development of urban and rural results is low, and the income and living consumption gap of urban and rural residents is large. In the future, the vitality of development in rural areas should be fully explored to promote the sharing of development results among urban and rural residents. From the perspective of fiscal surplus, related scholars proposed that citizens with the same income can enjoy the same fiscal treatment in different regions [15]. The realization of equalization of fiscal surplus is conducive to not only maintaining fairness and justice in the process of economic development but also promoting financial resources between regions. Researchers conducted a performance evaluation of public education in Oklahoma and found that the results produced by different estimation methods have certain deviations, but they all indicate that the government's fiscal expenditure has not always maintained high efficiency $[16,17]$. There are still investments in certain areas. Issues such as output mismatch, idle resources and waste, and the regional socioeconomic development are the decisive factors for low efficiency. Relevant scholars have constructed an evaluation index system that includes 16 indicators from five perspectives: infrastructure, basic education services, public employment services, public medical services, and social security [18]. The Theil index method is used to measure the degree of equalization of public services in various provinces. However, foreign scholars' research on public services mainly focuses on the theoretical aspects [19]. The evaluation of the level of public service equalization is somewhat single, and representative indicators are rarely selected for measurement and analysis from an overall perspective. The research fields mainly focus on education and medical care. In this respect, the research on influencing factors also focuses on single influencing factors such as public financial expenditure and investment and ignoring the effect of multiple factors [20]. The research on the relationship with economic growth also mainly selects fiscal expenditures in various fields $[21,22]$. The research direction is slightly single, and quantitative analysis is relatively rare.

The technical contributions of this article can be summarized as follows.

First, we combine immune algorithm with genetic algorithm and introduce the construction process of improved immune genetic algorithm applied to the coordinated development of China's regional economy, including the design of adaptive crossover and mutation operators, the calculation of antibody affinity, the calculation of binding strength, the structure of selection probability, and the calculation of concentration.

Second, the improved immune genetic algorithm is realized, and the multiobjective forecasting problem of singlecycle remanufacturing supply chain logistics network is solved. The process of solving the multiobjective forecasting problem in a single-cycle remanufacturing supply chain is divided into two stages: the first stage is to determine the layout of various facilities in the remanufacturing supply chain system and at the same time determine the logistics facilities in the remanufacturing supply chain system, and the fixed operating cost is determined in the first genetic algorithm; the second stage is to determine the specific types of logistics through the second genetic algorithm on the basis of determining the layout of various logistics facilities in the first genetic algorithm and at the same time determining the transportation cost of the remanufacturing supply chain logistics network and the environmental impact processing cost.

The rest of this article is organized as follows. Section 2 analyzes the relevant theories of the coordinated development of regional economy. Section 3 designs an improved immune genetic algorithm. In Section 4, a case analysis is carried out. Section 5 summarizes the full text. 


\section{Theoretical Analysis of the Coordinated Development of Regional Economy}

2.1. Regional Logistics-Economic-Environmental System. The regional logistics-economy-environmental system is a dynamic organic whole coupled by the three subsystems of regional logistics, regional economic system, and regional environment, in order to realize the composite system of the coordinated development of regional logistics, economy, and environment. The specific architecture is shown in Figure 1.

The regional logistics subsystem is composed of logistics facilities, logistics enterprises, information, funds, personnel, and other dynamic elements in the radiation range of a specific area and its associated space. It aims to improve logistics efficiency and achieve synergy.

The regional economic subsystem refers to a combination of various structural elements related to economic development in a specific region, and a functional structure combination with unique regional characteristics formed in various forms. The regional economic subsystem covers all sectors of the regional national economic system. It is also a complex and open dynamic system that is connected with the environment and resources within and outside the region in terms of production, exchange, distribution, and consumption. The main body of the operation of the regional economic subsystem is various types of enterprises, that is, the enterprises that generate logistics demand in the first, second, and tertiary industries. It is the demand system of the entire regional logistics.

\subsection{Coupling of Regional Logistics-Economic-Environmental} Systems. The various components in the regional logisticseconomy-environmental system continuously influence each other to promote the coupling and evolution of the system. The regional logistics subsystem, economic subsystem, and environmental subsystem cooperate closely to meet the needs of social logistics and the harmonious development of the economic environment [23-25]. They are mutually independent and interact with each other, forming a triple helix structure with the cross-influence of three forces. In this structure, it does not emphasize a certain subsystem as the main body but emphasizes the cooperative relationship of the three. The three can become dynamic leaders in different periods. The three are mutually beneficial, interact, and overlap each other. When economic development is lagging behind, the economic subsystem is the dominant and development is given priority, and logistics and the environment are second. For example, when China had just reformed and opened up, priority was given to economic development; when the economy develops to a certain stage, the lagging logistics system becomes a restrictive factor for economic development. At this time, the logistics priority development strategy should be implemented; when economic development causes huge damage to the environment, the environment has become the main restricting factor for the development of complex systems. These three constitute a cyclic, spiral-like coupling mode.
The coupling process of regional logistics-economics-environmental system is shown in Figure 2.

In the process of system coupling, each element continuously interacts with effectors, detectors, rule bases, and information bases. If there is information that matches the elements, they take corresponding actions and give feedback. Through continuous learning, adaptation, adjustment, and improvement, the functional structure and operation mechanism of each subsystem are coupled and perfected. The elements of the subsystem screen the information input from the outside and input the information into the system through the detector. The detector is used to filter information and filter information that meets the specific requirements of the system.

The information database is a database composed of a large amount of historical data and information, as well as experience and case information. It provides a comparative reference for the information input by the outside world, such as regional logistics demand and supply over the years, economic and industrial structure, and environmental information. The behavior of each subsystem and component elements in the regional logistics-economic-environmental system is determined by a series of rules. Some rules are used to filter environmental information and act on detectors. Some rules act on effectors and promote actions in the system. The rules of all actions constitute the system's rule base.

Through the screening of materials, information, and energy input from the outside world, each component element is adaptively studied and based on the data and information stored in the information database, and selforganizing operations are adopted to complete the internal coupling of the system, thereby forming a well-organized and well-functioning regional logistics-economic-environmental system. The external manifestation is the efficient operation of logistics, sustainable economic development, and harmonious coexistence of the environment.

\subsection{Coupling Development Process of Regional Logistics-} Economy-Environmental System. According to the theory of dissipative structure and system evolution mechanism, the coupling development of regional logistics-economic-environmental system can be roughly divided into four stages: the budding stage, the forming stage, the running-in stage, and the coordination stage, as shown in Figure 3.

In the early stage of the formation of the regional logistics-economy-environmental system, there is almost no connection between regional logistics, economy, and the environment. Regional logistics is in its infancy and has little contribution to economic growth; its impact on the environment is minimal. At this stage, the system is in a state of chaos and disorder with high entropy. If the system is in a relatively closed environment at this time, it cannot absorb negative entropy from the outside world.

With the gradual development of the economy and the growth of the logistics industry, its role in the regional economy has gradually manifested. The regional logistics- 


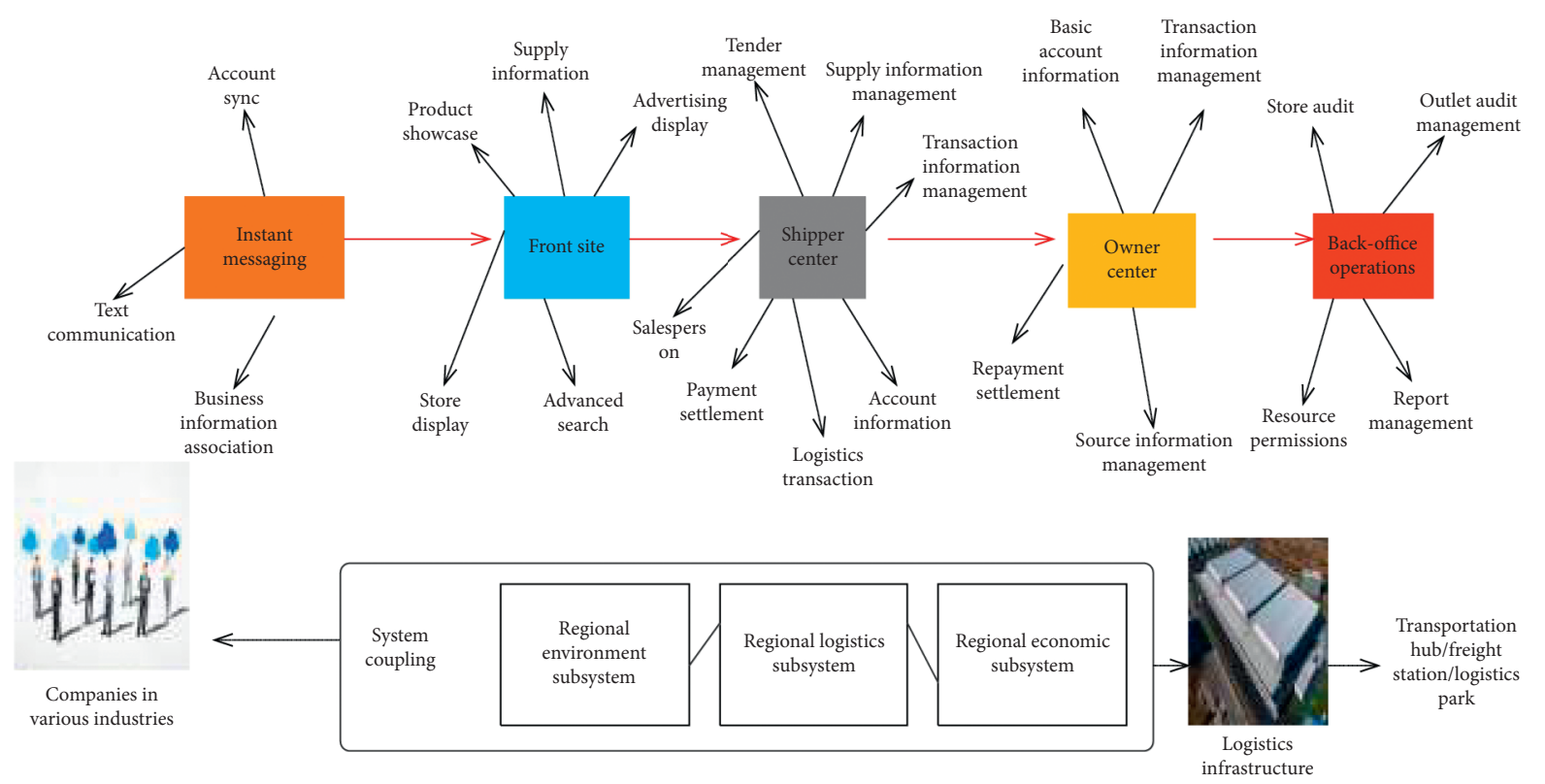

FIgURE 1: Regional logistics-economic-environmental system architecture.
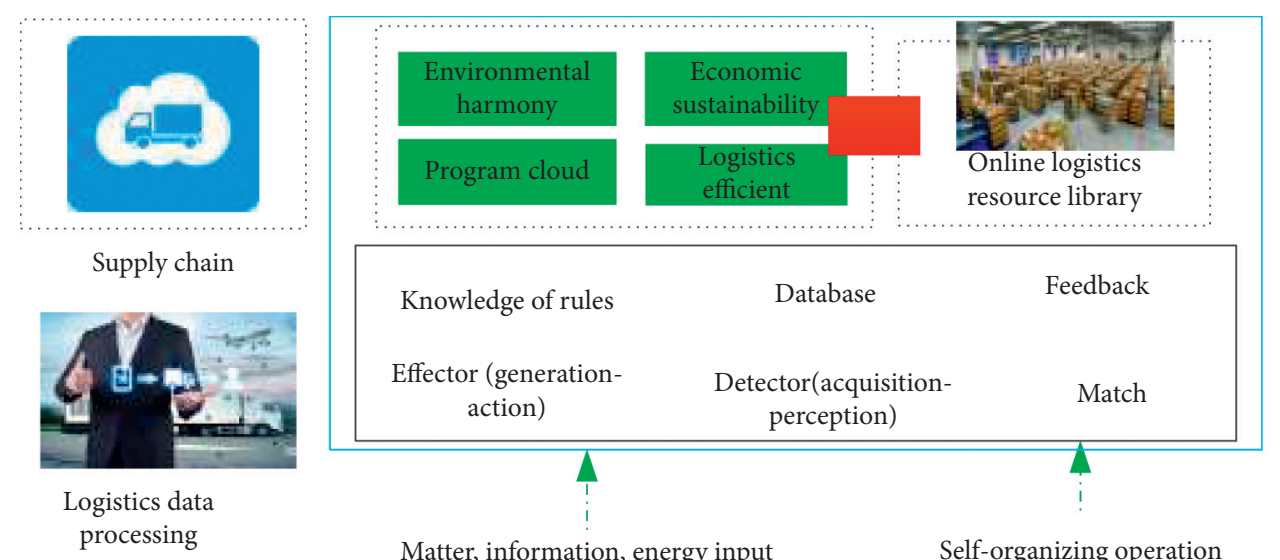

Matter, information, energy input

Self-organizing operation

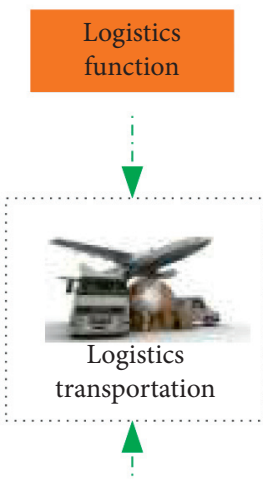

Third-party logistics integrator

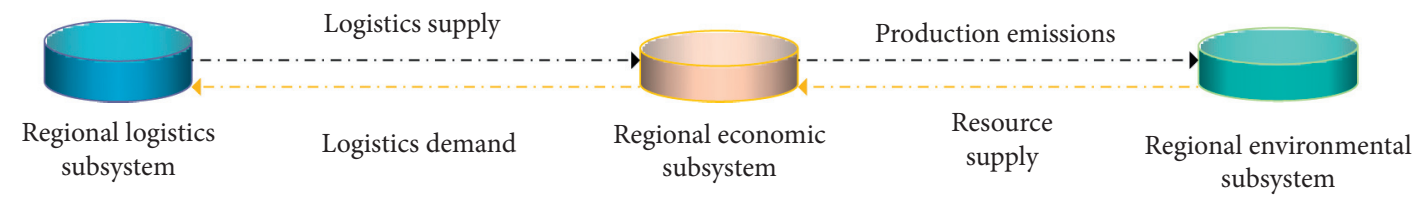

FIGURE 2: System coupling process.

economy-environmental system has begun to enter the second stage, the formation stage. But, at this time, the system has not yet formed an orderly structure, the internal coupling organization has not yet been established, and the various components are in a low degree of coupling. The specific manifestation is that the development of the regional logistics industry is chaotic, in a state of disorderly competition, industry policy support and legal supervision are not in place, and the entire industry is in a state of disorderly and free development.

With the rapid development of the logistics industry, its role in economic growth has become more and more important, and the government has paid more and more attention. The implementation of various policies and measures to support the development of the logistics industry has gradually achieved results, and the disordered state of the logistics industry has begun to change. The industry began to develop in a stable and orderly direction. The subsystems of the regional logistics-economyenvironmental system began to support and cooperate with each other, and various governments and regulations began to play a substantial role, but they were still in the running-in stage and failed to fully function, and the system entered a state of moderately coupled development. 


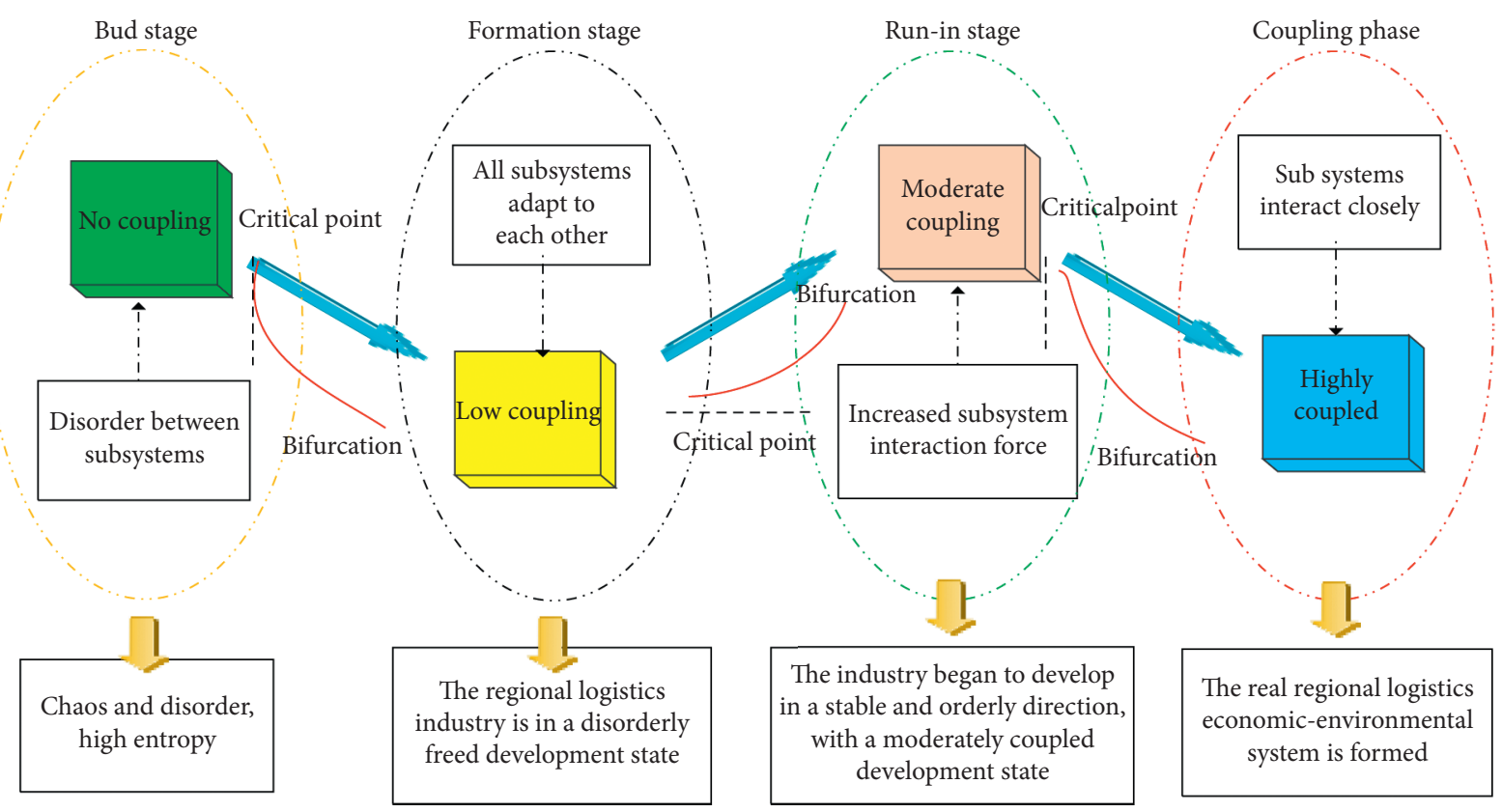

FigURE 3: Development stage of regional logistics-economic-environmental system coupling.

Due to the differences between subsystems, the ability of subsystems to obtain resources from the outside world must be different, and the effectiveness of resources leads to inevitable competition. When the system obtains energy and information from the outside world, the competition between subsystems leads to the improvement of the system's production mode and production efficiency and at the same time promotes the evolution of the regional logistics-economicenvironmental system in an orderly direction. Therefore, competition is the internal driving force of system evolution and the prerequisite and condition for collaboration.

There are a large number of multiple nonlinear relationships among the elements in the regional logistics-economicenvironmental system. Therefore, regional logistics, economy, and environment are interrelated in each production link. The regional logistics subsystem provides logistics services for the development of the regional economic subsystem; the regional economic subsystem provides financial support for the construction of the regional logistics subsystem; the regional environmental subsystem provides policy, technology, and resource support for the development of regional logistics and economic subsystems. Therefore, collaboration makes the subsystems support each other, where the structure is closer, and promotes the development of the system to a higher level. Therefore, the internal condition for the coupled development of the regional logistics-economy-environmental system is the internal competition and coordination mechanism of the system, which jointly promote the system to achieve an orderly structure and develop to a higher level, thereby achieving coupled development.

\section{Improved Immune Genetic Algorithm Design}

3.1. Immune Genetic Algorithm. Immune genetic algorithm is an improved genetic algorithm based on the organism's own immune regulation mechanism. Its essence is to add biological immune mechanism to the standard genetic algorithm and make it merge. It not only has the advantages of high parallel computing ability, global optimization ability, and stability of traditional genetic algorithm but also can effectively improve the search accuracy and local convergence speed of traditional genetic algorithm due to the addition of biological immune mechanism in the algorithm. Since the immune genetic algorithm can improve the accuracy and speed of the design, thereby saving labor time and labor cost, the practical application of the algorithm is increasing.

Immune genetic algorithm is an improved modern optimization algorithm designed to simulate the immune characteristics of the organism's own immune system in biology. Therefore, the immune genetic algorithm basically retains the basic characteristics and structure of the standard genetic algorithm. At the same time, the affinity calculation and the structure of the immune memory unit are added to the traditional genetic algorithm to improve the algorithm and make the optimization result more accurate.

The immature convergence phenomenon of genetic algorithm is caused by the poor population diversity caused by the uneven distribution of solution groups in the process of genetic evolution; the poor local search ability is because the operator designed by the genetic algorithm is for global search $[26,27]$. Therefore, the improvement of genetic algorithm should be aimed at improving the diversity of the population and improving the local search ability.

Immune genetic algorithm (IGA) is also a way to improve genetic algorithm. It is a genetic algorithm with immune function, which is a typical hybrid intelligent calculation method. It not only has the advantages of genetic algorithm but also absorbs the advantages of immune algorithm, showing better superiority in many aspects 
[28-30]. Compared with the genetic algorithm, it has increased the antigen recognition function, the extraction, and inoculation of the vaccine, has a memory function, does not have very complicated operations, takes into account the algorithm search and operation speed, and maintains the applicability and robustness of the genetic algorithm. The immune genetic algorithm is also the same as the immune algorithm. The problem to be solved is used as the antigen and the solution of the problem to be solved is used as the antibody. The difference lies in the use of genetic operators in the immune genetic algorithm. The algorithm not only retains the search characteristics of genetic algorithm and avoids the shortcomings of low efficiency of genetic algorithm local search but also avoids premature phenomenon to a large extent.

Vaccine is the relatively essential characteristic information extracted from the more or less experience accumulated by people for the problem to be solved. The extraction of vaccine can be analyzed in detail for specific problems and selected according to the actual characteristics of the problem to be solved, such as the value range of the web thickness of the crane main beam, or the restriction relationship between the web and the upper cover. A good selection of immunization vaccines has a good guide to predict the direction of the target solution and has a significant effect on accelerating the convergence speed of the algorithm.

After calculating the fitness of an individual, if the fitness value is satisfactory, the individual can be used as a candidate for the optimal solution; if the fitness value is very low, the individual is vaccinated to change some genes in its gene sequence, so that individuals can have better fitness. It can be seen that vaccination is to improve the fitness of the population.

\subsection{Design of Improved Immune Genetic Algorithm}

3.2.1. Affinity Calculation. There are usually four methods for calculating affinity, which are $R$ continuous position method, Euclidean distance method, Hamming distance method, and information entropy measurement method. The information entropy measurement method can mainly be used to measure the diversity of antibodies, so this article uses the information entropy measurement method to calculate the affinity.

The formula for calculating the affinity $\mathrm{D}_{a, b}$ between any two antibodies $a$ and $b$ is

$$
D_{a, b}=\frac{1}{E(2)+1} \text {. }
$$

In the formula, $E(2)$ is the average information entropy of antibody $a$ and antibody $b$. The larger the value of $D_{a, b}$, the more similar the antibody $a$ and the antibody $b$.

Similarly, the affinity $\mathrm{Dy}_{b, c}$ between antibody $b$ and antigen $c$ can be calculated by the following formula:

$$
D y_{a, b}=\frac{1}{E_{b, c}+1} \text {. }
$$

The affinity between antibodies is calculated as follows:

$$
D y_{b, c}=\frac{1}{H_{b, c}+1} \text {. }
$$

In the formula, $H_{b, c}$ represents the binding strength of antibody $b$ and antibody $c$.

3.2.2. Adaptive Crossover and Mutation Probability. Based on the existing immune genetic algorithm, this paper designs an adaptive immune genetic algorithm. The algorithm uses the theory of information entropy to set the crossover probability $p_{c}$ and mutation probability $p_{m}$ so that it can be autonomous according to the degree of fitness adjust.

According to the information entropy theory, the information entropy of the $j$ th gene on the chromosome can be calculated as follows:

$$
E_{j}(M)=-P_{i j} \cdot \lg P_{i j}+\Sigma_{i=0}^{s-1} .
$$

The average information entropy of $M$ antibodies is calculated by the following formula:

$$
E(M)=\frac{\sum_{j=0}^{N-1} E_{j}(M-1)}{N} .
$$

The population similarity is

$$
B(M)=\frac{1}{E(M)-1} .
$$

In the formula, $B(M)$ represents the similarity degree of the population as a whole, and the value range is $(0,1)$. The higher the similarity of the population, the greater the value of $B(M)$.

Crossover probability $p_{c}$ and mutation probability $p_{m}$ are the two main parameters in the immune genetic algorithm. Setting too large or too small will affect the entire operation process of the algorithm and may cause the convergence speed to be too slow or not to converge, making it difficult to obtain the optimal value. The introduction of the adaptive concept can make the two parameters $p_{c}$ and $p_{m}$ change with the fitness of the population. When the fitness of individuals in the population shows a tendency to converge to one value, $p_{c}$ and $p_{m}$ increase accordingly; and when individual fitness is distributed to many values, $p_{c}$ and $p_{m}$ decrease correspondingly.

3.2.3. Select Probability Calculation. The selection probability $p_{s}$ in the immune genetic algorithm is jointly determined by the fitness probability $p_{f}$ and the concentration probability $p_{d}$ and is calculated as follows:

$$
p_{s}=(1-a) \cdot p_{d}+a \cdot p_{f}
$$

The concentration probability $p_{d}$ is calculated as follows:

$$
p_{d}=\frac{\alpha-C_{i}+C_{\max }}{C_{\max }^{\beta}} .
$$


The selection probability $p_{s}$ calculated in this way not only ensures the promotion effect of the fitness value on the selection but also regulates and controls it through the effect of concentration, which can inhibit the concentration of similar antibodies and ensure the population diversity in the population.

3.2.4. Calculation of Concentration. Similar to affinity, concentration is also an important indicator of the diversity of antibody populations. In the immune system, when an antibody is stimulated by an antigen, the concentration of antibodies with high affinity increases, and when it reaches a certain level, it is inhibited. On the contrary, it promotes the increase of antibodies with low concentration. This situation is the promotion and inhibition of antibody production. The promotion and inhibition of antibodies maintain the diversity of the population. Concentration indicates how many antibodies are similar to a certain antibody within the threshold range in the antibody population. The concentration can not only reflect the degree of similarity between antibodies but also reflect the number of similar antibodies in the population. The addition of the concept of concentration introduces a concentration adjustment mechanism based on the original fitness calculation. For any antibody $w$, the following formula can be used to calculate its concentration $C_{w}$ :

$$
C_{w}=\frac{\left|\left[v \longrightarrow X \mid D_{b, c}<\lambda\right]\right|}{M} .
$$

The specific process of predictive design based on adaptive immune genetic algorithm is shown in Figure 4 .

\section{Examples and Analysis}

4.1. Numerical Examples. In the single-cycle remanufacturing supply chain system environment, it is assumed that there are 4 manufacturing/remanufacturing integrated factory and 20 consumption areas. Distribution centers and recycling centers are set up at 20 candidate locations on the logistics network, among which 3 distribution centers and 3 recycling centers are set up at these candidate locations.

The unit costs $\mathrm{c} 1$ and $\mathrm{c} 2$ of forward supply chain logistics transportation are both 1 yuan/piece. $\mathrm{km}$. Considering that the use of forward logistics distribution vehicles to transport and recycle old parts can save transportation costs, the unit costs of reverse supply chain logistics and transportation $\mathrm{cr} 1$ and $\mathrm{cr} 2$ are both 0.76 yuan $/$ piece $\cdot \mathrm{km}$. The fixed operating cost of the integrated factory is 102,000 yuan, and the cost of producing products is 302 yuan. The operating costs of sales and recycling centers at 20 alternative points are shown in Figure 5.

If an integration center for product distribution and waste product recycling is set up at the same alternative location, the operating expenses of this integration center will be compared with the total operating expenses of the distribution center and recycling center at this alternative location, and the total expenses can be saved $\beta=0.2$.

The unit selling price $p$ of the distribution center is 1000 yuan, and the unit recycling price pr of the recycling center is
150 yuan. The unmanufactured residual value e 1 of the unit of the integrated manufacturing plant is 100 yuan. The unusable residual value e 2 of the unit in the recycling center is 120 yuan. The recyclability of recycled waste products in the recycling center is $\beta 2=0.3$. The remanufacturing rate of the integrated manufacturing plant is $\beta 1=0.4$. The unit cost pe for dealing with environmental impact is 500 yuan/unit. The environmental impact coefficients $\sigma 1$ and $\sigma 2$ are 0.12 units. The harmful environmental impacts h1 and h2 per unit distance caused by transportation are 0.013 unit and 0.024 unit, respectively.

$\mathrm{D} n$ is expressed as the random market demand in consumption area $n$. The maximum market capacity is $Q n$, and the market demand price elasticity coefficient $\alpha$ is 0.14 . The largest proportion of market demand capacity in each consumption area is shown in Figure 6.

It is assumed that the production capacity of the integrated manufacturing/remanufacturing factory and the remanufacturing capacity of waste products are unlimited. We set up the respective processing capacity of the distribution center and recycling center in 20 alternative locations, as shown in Figure 7.

In the remanufacturing closed-loop supply chain system, forward logistics and reverse logistics are integrated. Therefore, the route distance between various types of facilities is consistent. The distance between 4 integrated factories and 20 alternative locations is shown in Figure 8.

Customer satisfaction in the remanufacturing supply chain system is determined by the product delivery time. In the consumption area, the customer can deliver the product within 6 hours after placing the product demand order, which is the best time window for customer satisfaction, $t \in$ $[0,6]$. The product demand order can be delivered within 2 days, which is acceptable to the customer, $t \in[0,48]$. After 2 days, the customer will refuse to accept the goods.

\subsection{Calculation Example Results}

4.2.1. Single-Cycle Remanufacturing Supply Chain Logistics Network under Dual Random Environment. After solving by an improved immune genetic algorithm, the final optimal solution of the remanufacturing supply chain is obtained. Distribution centers are built in alternative locations 1, 4, and 5 , and recycling centers are built in alternative locations 1,3 , and 6. An integration center can be built on. Distribution center 1 supplies products for consumption area 2 and consumption area 3, distribution center 4 supplies products for consumption area 4 and consumption area 5, and distribution center 5 supplies products for consumption area 1 . Recycling center 1 provides service for recycling waste products in consumption area 3 and consumption area 5 , recycling center 3 provides service for recycling waste products in consumption area 4, and recycling center 6 provides service for recycling waste products in consumption area 1 and consumption area 2.

For the remanufacturing supply chain logistics network prediction problem in a dual random environment, the cluster prediction solution simulation of genetic algorithm 


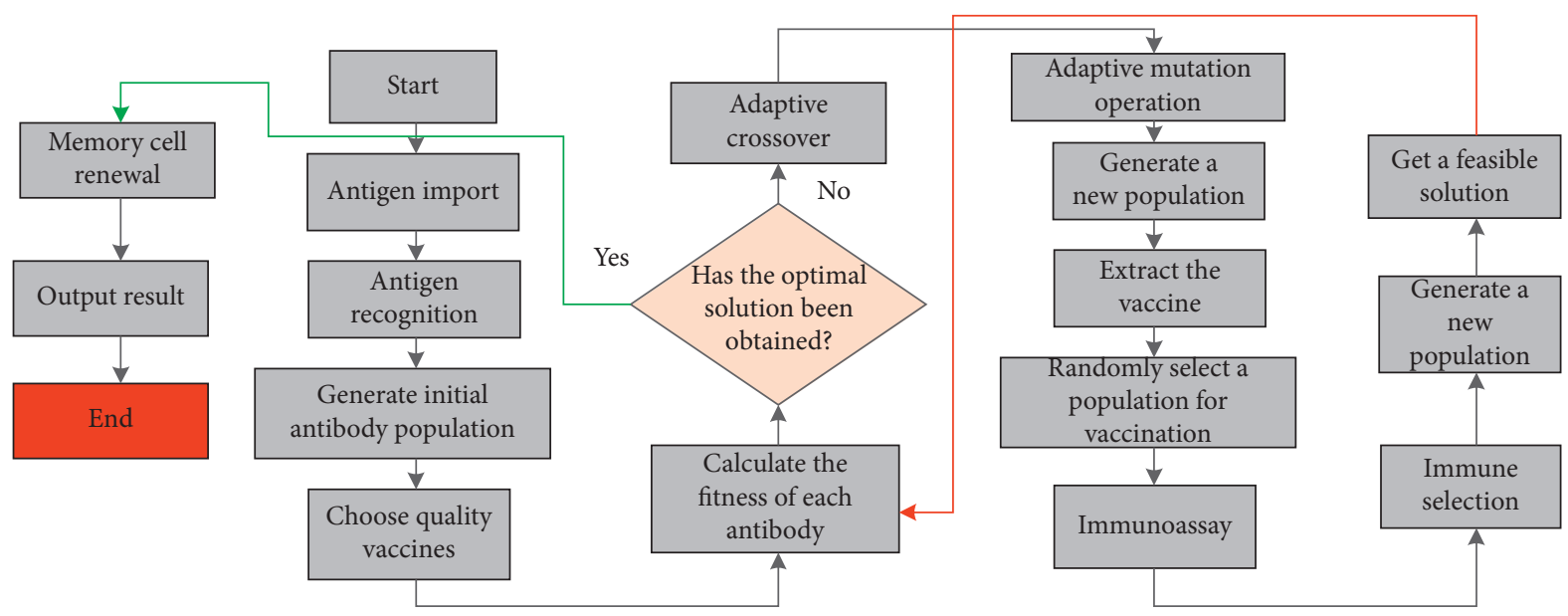

Figure 4: Predictive design process based on adaptive immune genetic algorithm.

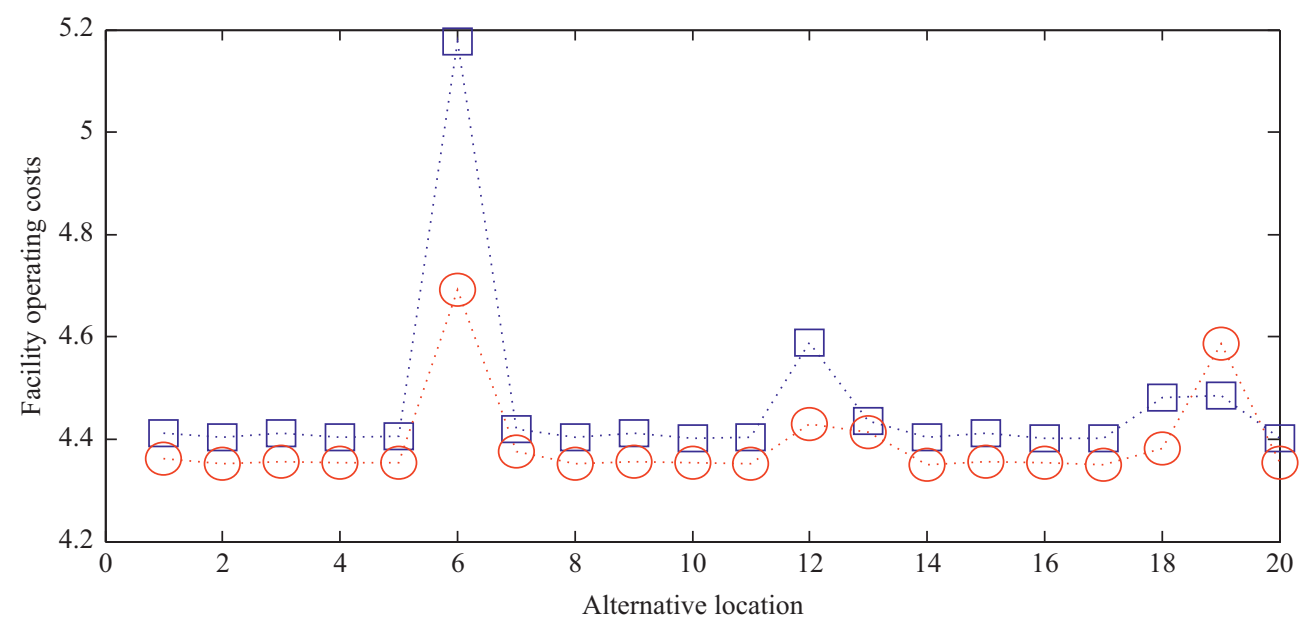

Distribution center operating costs

. Recycling center operating costs

FIgURE 5: Operating costs of various types of logistics facilities at alternative locations.

and improved immune genetic algorithm is shown in Figure 9.

It can be seen from the convergence curves of the two genetic algorithms that the improved immune genetic algorithm is better than the genetic algorithm and can be closer to the optimal solution of the prediction model under the same calculation example. This is because the improved immune genetic algorithm processes the decision-making variables of the prediction model hierarchically and takes the establishment variables of various types of logistics facilities in the supply chain system as the first decision-making variables in the prediction process; the logistics distribution status variables among the logistics facilities are the second. The decision variables in the reprediction process are based on the decision variables in the first reprediction process. It can avoid the disadvantages of too long coding digits of decision variables and complicated operations of crossover and mutation operators. From the aspect of predicting the convergence speed, the improved immune genetic algorithm has improved compared with the genetic algorithm.

4.2.2. Order Cycle Remanufacturing Supply Chain Logistics Network under Option Contract Mechanism. After solving by an improved immune genetic algorithm, the final optimal solution of the remanufacturing supply chain is obtained. Distribution centers are built in alternative locations 2, 4, and 5 , and recycling centers are built in alternative locations 1,2 , and 3. An integration center can be built on alternative site 2. Distribution center 2 supplies products for consumption area 2 and consumption area 3 , distribution center 4 supplies products for consumption area 4 and consumption area 5 , and distribution center 5 supplies products for consumption area 1 . Recycling center 1 provides service for recycling waste products in consumption area 2, recycling center 2 provides recycling service for waste products 


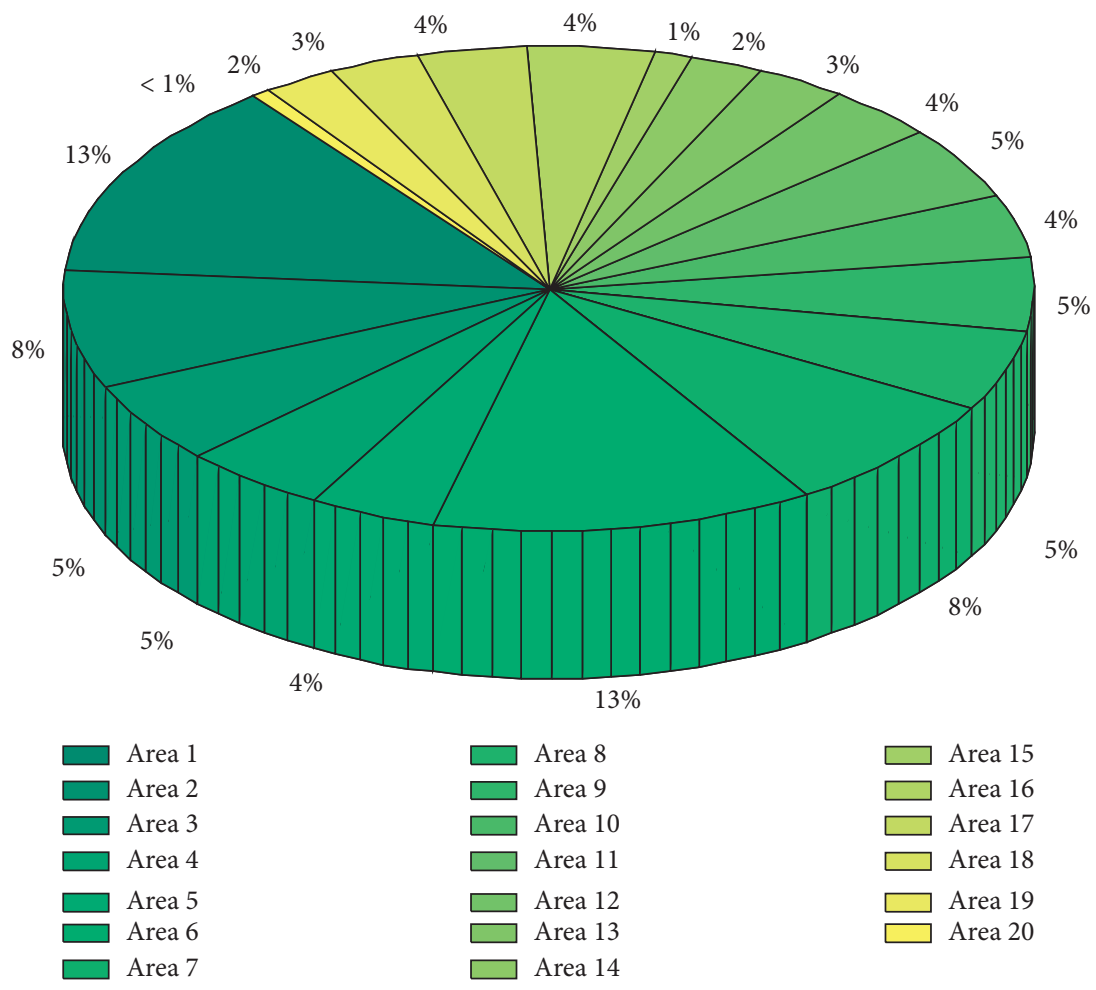

Figure 6: Proportion of the largest market demand capacity $Q n$ in each consumption region.

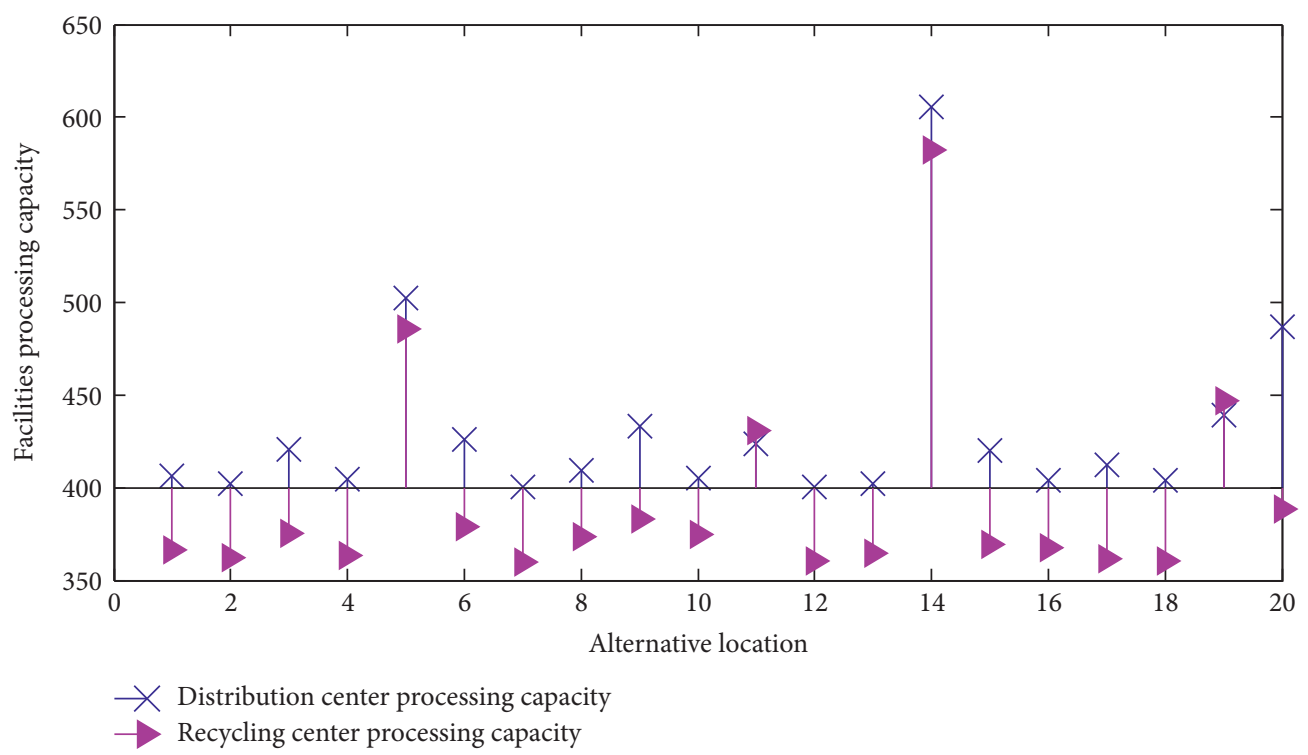

FIgURE 7: Processing capacity of various types of logistics facilities at alternative locations.

in consumption area 3 and consumption area 4 , and recycling center 3 provides service for recycling waste products in consumption area 1 and consumption area 5. Finally, the optimal value of the remanufacturing supply chain system under the dual random environment is 619 thousand yuan.

The forecasting problem of the logistics network of the single-period remanufacturing supply chain under the option contract mechanism and the cluster forecasting simulation of the improved immune genetic algorithm is shown in Figure 10.

By comparison with the results of the previous calculation examples, it can be concluded that, under ideal conditions, that is, the random product market demand function value $\mathrm{H}$ is equal to the actual product market demand $h$, the logistics network forecasting problem using 


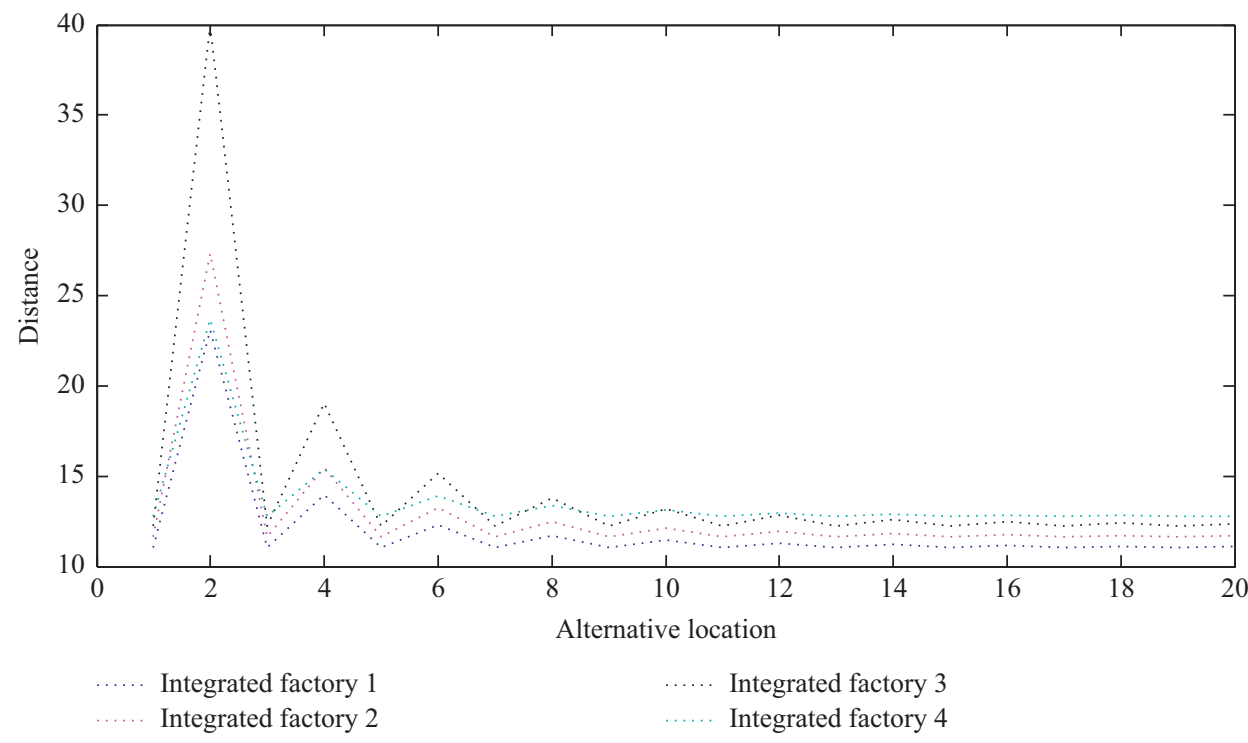

FIgURE 8: Distance from integrated factory to alternative location.

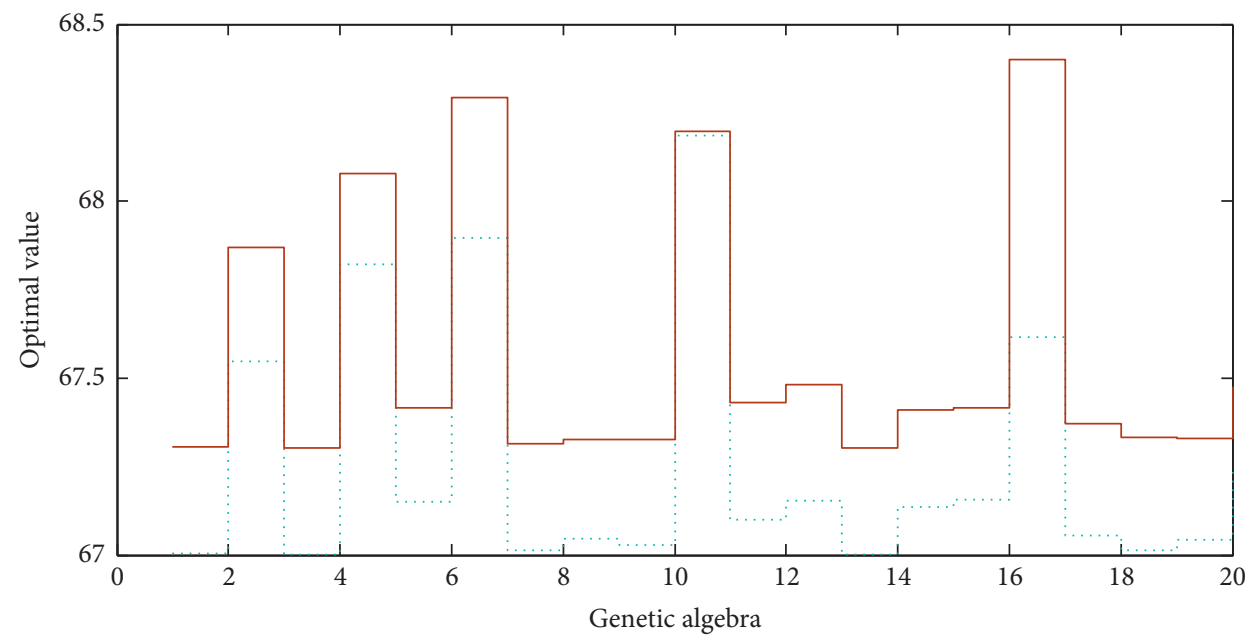

Genetic algorithm

_ Improved immune genetic algorithm

FIgURE 9: Cluster prediction solution simulation diagram of genetic algorithm and improved immune genetic algorithm.

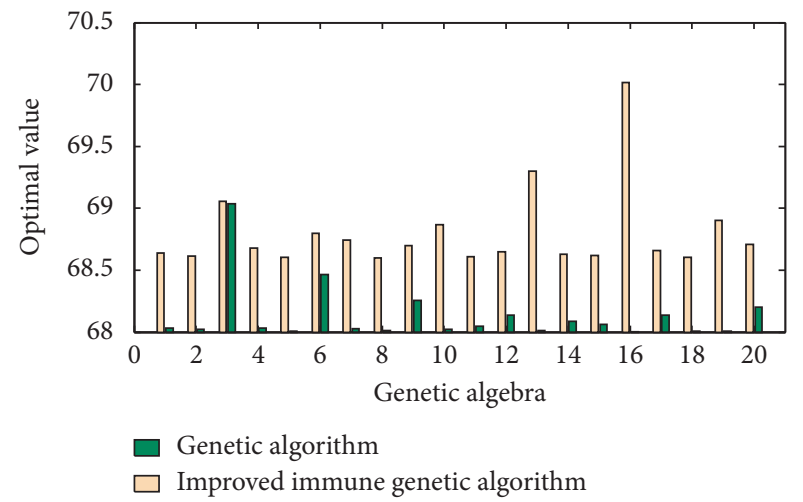

FIGURE 10: Simulation diagram of clustering prediction solution for logistics network problem of single-period remanufacturing supply chain under option contract mechanism. the option contract mechanism is better than that in the double random environment. The profit of network forecasting problems is less. This is because part of the total profit predicted by the logistics network under the option contract mechanism is used as additional maintenance funds to control the uncertain market demand.

In the multiobjective forecasting model of the remanufacturing supply chain logistics network based on the option contract mechanism, the closer the actual product market demand $h$ is to the number of product prepurchase options, the greater the operating profit of the logistics network of the remanufacturing supply chain system is.

In summary, the single-period remanufacturing supply chain logistics network multiobjective forecasting model based on the option contract mechanism is more suitable for the changing and uncertain product market demand 
environment and most of the remanufacturing supply chain logistics network forecasting models. The multiobjective forecasting model of logistics network with dual random environment is proposed, which is only suitable for a certain point of uncertain product market demand.

\section{Conclusion}

This paper studies the related theories of genetic algorithm and artificial immune algorithm. On this basis, the two algorithms are combined, the genetic algorithm is used as the basic framework, and the operators and selection methods of artificial immune algorithm are integrated. The crossover probability and mutation probability are improved, and the adaptive crossover probability and mutation probability formula are innovatively designed by using the adaptive concept. Compared with the fixed value of the basic immune genetic algorithm, the introduction of the adaptive concept can be used for optimization. The process is intelligently adjusted, which improves the optimization speed and increases the diversity of antibody populations. The logistics network forecast of the single-cycle remanufacturing supply chain system should consider not only the forward sales logistics network forecast but also the reverse recycling logistics network forecast. Taking the maximization of logistics network operation income, the maximization of customer satisfaction, and the minimization of environmental impact as the forecasting goals, a multiobjective forecasting model of remanufacturing supply chain logistics network under a dual random environment is established. The option contract mechanism is used to effectively control the market demand for uncertain products, and a multiobjective forecasting model of the remanufacturing supply chain logistics network based on the option contract mechanism is established. An improved immune genetic algorithm is designed to solve the prediction model, and the prediction model and algorithm are verified through examples, and their effectiveness is analyzed.

\section{Data Availability}

The data used to support the findings of this study are available from the corresponding author upon request.

\section{Conflicts of Interest}

The author declares that there are no conflicts of interest reported in this paper.

\section{References}

[1] Z. C. Ruhe, D. A. Low, and C. S. Hayes, "Polymorphic toxins and their immunity proteins: diversity, evolution, and mechanisms of delivery," Annual Review of Microbiology, vol. 74, no. 1, pp. 497-520, 2020.

[2] M. Gordan, Z. B. Ismail, H. Abdul Razak, K. Ghaedi, and H. H. Ghayeb, "Optimization-based evolutionary data mining techniques for structural health monitoring," Journal of Civil Engineering and Construction, vol. 9, no. 1, pp. 14-23, 2020.
[3] W. Hua, H. Ye, J. Y. Rau et al., "Dynamic prediction of uneven-aged natural forest for yield of Pinus taiwanensis using joint modelling," Environmental Monitoring and Assessment, vol. 192, no. 4, pp. 1-13, 2020.

[4] Q. Zhou, H. Gao, Y. Zhang et al., "A chromosome-level genome assembly of the giant grouper (Epinephelus lanceolatus) provides insights into its innate immunity and rapid growth," Molecular Ecology Resources, vol. 19, no. 5, pp. 1322-1332, 2019.

[5] X. Ye, B. Chen, L. Jing, B. Zhang, and Y. Liu, "Multi-agent hybrid particle swarm optimization (MAHPSO) for wastewater treatment network planning," Journal of Environmental Management, vol. 234, pp. 525-536, 2019.

[6] M. Gulyaeva, F. Huettmann, A. Shestopalov et al., "Data mining and model-predicting a global disease reservoir for lowpathogenic Avian Influenza (A) in the wider pacific rim using big data sets," Scientific Reports, vol. 10, no. 1, pp. 1-11, 2020.

[7] A. Maslova, R. N. Ramirez, K. Ma et al., "Deep learning of immune cell differentiation," Proceedings of the National Academy of Sciences, vol. 117, no. 41, pp. 25655-25666, 2020.

[8] H. Bo, X. Niu, and J. Wang, "Wind speed forecasting system based on the variational mode decomposition strategy and immune selection multi-objective dragonfly optimization algorithm," IEEE Access, vol. 7, pp. 178063-178081, 2019.

[9] B. Han, H. Chen, Y. Yao et al., "Genetic and non-genetic factors associated with the phenotype of exceptional longevity \& normal cognition," Scientific Reports, vol. 10, no. 1, pp. 1-15, 2020.

[10] J. Chen, B. Lu, and L. Hao, "Research on optimal collaborative method for microgrid environmental and economic dispatch in grid-connected mode," International Journal of Simulation and Process Modelling, vol. 14, no. 6, pp. 513-523, 2019.

[11] A. Zou, K. Nadeau, P. W. Wang et al., "Accumulation of genetic variants associated with immunity in the selective breeding of broilers," BMC Genetics, vol. 21, no. 1, pp. 1-14, 2020.

[12] D. Freites-Núñez, A. Baillet, L. Rodriguez-Rodriguez et al., "Efficacy, safety and cost-effectiveness of a web-based platform delivering the results of a biomarker-based predictive model of biotherapy response for rheumatoid arthritis patients: a protocol for a randomized multicenter single-blind active controlled clinical trial (PREDIRA)," Trials, vol. 21, no. 1, pp. 1-15, 2020.

[13] D. Zeng, Y. Gao, Y. Hu, and J. Liu, "Optimization control for the coordinated system of an ultra-supercritical unit based on stair-like predictive control algorithm," Control Engineering Practice, vol. 82, pp. 185-200, 2019.

[14] A. Zhu, S. Yang, Q. Li et al., "Research on prediction of metro wheel wear based on integrated data-model-driven approach," IEEE Access, vol. 7, pp. 178153-178166, 2019.

[15] A. M. Degoot, E. S. Adabor, F. Chirove et al., "Predicting antigenicity of influenza A viruses using biophysical ideas," Scientific Reports, vol. 9, no. 1, pp. 1-10, 2019.

[16] V. S. Misakov, L. A. Tsurova, L. A. Yandarbayeva et al., "Certification of a regional economic complex as a highly effective tool for analysis and diagnostics of its development," Amazonia Investiga, vol. 8, no. 20, pp. 451-458, 2019.

[17] O. Roth, M. H. Solbakken, O. K. Tørresen et al., "Evolution of male pregnancy associated with remodeling of canonical vertebrate immunity in seahorses and pipefishes," Proceedings of the National Academy of Sciences, vol. 117, no. 17, pp. 9431-9439, 2020.

[18] J. Salas-Leiva, J. M. Mazón-Suástegui, A. Teles, and D. TovarRamírez, "Structure and predictive metabolic contribution of 
intestinal microbiota of Longfin yellowtail (Seriola rivoliana) juveniles in aquaculture systems," Molecular Biology Reports, vol. 47, no. 12, pp. 9627-9636, 2020.

[19] L. Huang, M. C. Zhou, and K. Hao, "Non-dominated immune-endocrine short feedback algorithm for multi-robot maritime patrolling," IEEE Transactions on Intelligent Transportation Systems, vol. 21, no. 1, pp. 362-373, 2019.

[20] W. Yu and W. Huafeng, "Neural network model for energy low carbon economy and financial risk based on PSO intelligent algorithms," Journal of Intelligent \& Fuzzy Systems, vol. 37, no. 5, pp. 6151-6163, 2019.

[21] N. Serrat, M. Guerrero-Hernández, A. Matas-Céspedes et al., "PI3K $\delta$ inhibition reshapes follicular lymphoma-immune microenvironment cross talk and unleashes the activity of venetoclax," Blood Advances, vol. 4, no. 17, pp. 4217-4231, 2020.

[22] J. S. Peters, N. Ismail, A. Dippenaar et al., "Genetic diversity in Mycobacterium tuberculosis clinical isolates and resulting outcomes of tuberculosis infection and disease," Annual Review of Genetics, vol. 54, no. 1, pp. 511-537, 2020.

[23] L. Xiong, K. Li, and L. Yang, "A parallel immune genetic algorithm for community detection in complex networks," International Journal of High Performance Computing and Networking, vol. 11, no. 3, pp. 242-250, 2018.

[24] J.-K. Rhee, Y. C. Jung, K. R. Kim et al., "Impact of tumor purity on immune gene expression and clustering analyses across multiple cancer types," Cancer Immunology Research, vol. 6, no. 1, pp. 87-97, 2018.

[25] F. Akdeniz, M. Biçil, Y. Karadede, F. E. Özbek, and G. Özdemir, "Application of real valued genetic algorithm on prediction of higher heating values of various lignocellulosic materials using lignin and extractive contents," Energy, vol. 160, pp. 1047-1054, 2018.

[26] R. R. Chowdhury, F. Vallania, Q. Yang et al., "A multi-cohort study of the immune factors associated with $\mathrm{M}$. tuberculosis infection outcomes," Nature, vol. 560, no. 7720, pp. 644-648, 2018.

[27] M. Duma and B. Twala, "Optimising latent features using artificial immune system in collaborative filtering for recommender systems," Applied Soft Computing, vol. 71, pp. 183-198, 2018.

[28] J.-S. Seo, J. W. Lee, A. Kim et al., "Whole exome and transcriptome analyses integrated with microenvironmental immune signatures of lung squamous cell carcinoma," Cancer Immunology Research, vol. 6, no. 7, pp. 848-859, 2018.

[29] H. Y. Li, C. Gui, and K. Xiao, "Simulation of multivariate scheduling optimization for open production line based on improved genetic algorithm," International Journal of Simulation Modelling, vol. 17, no. 2, pp. 347-358, 2018.

[30] C. Lopez, S. Tucker, T. Salameh, and C. Tucker, "An unsupervised machine learning method for discovering patient clusters based on genetic signatures," Journal of Biomedical Informatics, vol. 85, pp. 30-39, 2018. 